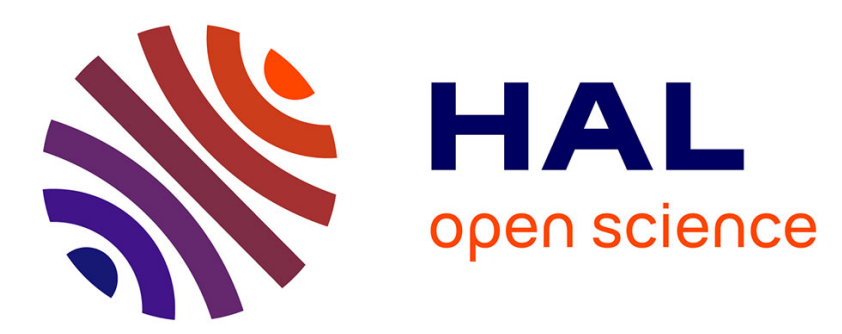

\title{
Value at Risk for confidence level quantifications in robust engineering optimization
}

\author{
Bijan Mohammadi
}

\section{To cite this version:}

Bijan Mohammadi. Value at Risk for confidence level quantifications in robust engineering optimization. Optimal Control Applications and Methods, 2013, 35 (6), pp.1-15. 10.1002/oca.2061 . hal-00845124

\section{HAL Id: hal-00845124 \\ https://hal.science/hal-00845124}

Submitted on 17 Oct 2013

HAL is a multi-disciplinary open access archive for the deposit and dissemination of scientific research documents, whether they are published or not. The documents may come from teaching and research institutions in France or abroad, or from public or private research centers.
L'archive ouverte pluridisciplinaire HAL, est destinée au dépôt et à la diffusion de documents scientifiques de niveau recherche, publiés ou non, émanant des établissements d'enseignement et de recherche français ou étrangers, des laboratoires publics ou privés. 


\title{
VALUE AT RISK FOR CONFIDENCE LEVEL QUANTIFICATIONS IN ROBUST ENGINEERING OPTIMIZATION
}

\author{
BIJAN MOHAMMADI
}

OPT. CONTR. APPLI. METH., 2013.

\begin{abstract}
We show how to introduce the Value at Risk (VaR) concept in optimization algorithms with emphasis in calculation complexity issues. To do so we assume known the PDF of the uncertainties. Our aim is to quantify our confidence on the optimal solution at low complexity without a sampling of the control space. The notion of over-solving appears naturally where it becomes useless to solve accurately near an optimum when the variations in control parameters fall below the uncertainties. Examples show the behavior of this VaR-based correction and link the approach to momentum-based optimization where the mean and variance of a functional are considered. The approach is then applied to an inverse problem with fluids with uncertainties in the definition of the injection devices. It is shown that an optimization problem with an admissible solution in the control space in the deterministic case can lose its solution in the presence of uncertainties on the control parameters which suggests that the control space itself should be redefined in such a situation to recover an admissible problem. This permits to evaluate the cost of making reliable a system which has been deterministically designed, but which has uncertain parameters. A shape optimization problem closes the paper showing the importance of including VaR information during the design iterations and not only at the end.
\end{abstract}

\section{INTRODUCTION}

Taking strongly into account the computational complexity constraint deeply impacts the way optimization problems are solved. In the past, we addressed global optimization from this view point $[2,6]$ and showed how to use a controllability result for a continuous second order dynamical system to improve the global search feature of existing algorithms.

In this paper, we would like to extend the optimization solution to situations where control parameters are uncertain but again with keeping the calculation complexity low. This means that any approach implying a sampling of the parameter space and calculation of probabilistic informations (momentums) is out of the table.

Taking into account manufacturing uncertainties is of utmost importance for a design procedure to be efficient. Indeed, it is impossible to make sure that the final product will exactly correspond to the design specifications. In shape optimization for instance, this uncertainty is rarely accounted for and shape design is performed for a given parameterization of the shape in a deterministic framework [8]. Recent works, however, show ways to include these. In [3], authors, after making a priori hypothesis on shape uncertainties, use proper orthogonal decomposition together with

Key words and phrases. Value at Risk, low complexity robust optimization, over-solving, Pareto front, inverse problems, CFD, reliability, uncertainty. 
sparse grid sampling [4] to reduce the probability space and a polynomial chaos propagation method [5] to evaluate the effect of the uncertainties on the functional in a nonintrusive way. Minimization can then be performed involving different functional momentums. This approach is suitable for industrial applications as manufacturing uncertainties are usually known through probability density functions which often comes as characteristics of the product manufacturing process. They can also be a posteriori identified through data assimilation in probability density functions from observations of manufactured devices $[9,11,10]$. However, in this work, we would like to avoid the use of reduced order modelling and separation between random and deterministic behaviors which is at the core of most uncertainty quantification analysis in literature.

1.1. SIMULATION UNDER UNCERTAINTY. Consider the following simulation chain linking a control parameter, its random perturbation (known through a probability density function), a state variable and a cost function:

$$
x \rightarrow \tilde{x}=x+\varepsilon(x) \rightarrow u(\tilde{x}) \rightarrow J(u(\tilde{x})) .
$$

This is an important situation: the place where randomness appears is well identified. Indeed, if $\varepsilon(x)=0$ the simulation is fully deterministic. It would then be interesting if one can keep the simulation and design processes deterministic and, still, somehow take into account these random perturbations during design. One classical approach is to propagate the randomness into the whole chain and get a noisy functional. And then assimilate this functional by a low-complexity model and design for this new functional $[3,8,12]$. This approach demands for a priori regularity hypothesis for the reduced-order model and trust regions definition. We will show however on a simple example that despite this approach is widely used, it can produce unsuitable results. Another possibility is to use Monte Carlo simulations and obtain the momentum of our noisy functional which then permit for momentum-based optimization. This, as we said, is not suitable in term of calculation complexity. We will compare our approach to this alternative on an example.

So the question is how to introduce the a priori information on $\varepsilon(x)$ into the optimization algorithm. We propose to combine the concept of value at risk and the knowledge of the sensitivity of the functional with respect to the control parameters. Our aim is indeed to have the gradient of the functional defined and evaluated as in the deterministic case where enough regularity is assumed available for this purpose. Eventually, this permits to quantify our confidence on the optimal solution without any sampling of the control space. Something which is important if one would like to keep the complexity low.

To make the approach efficient, we need to use existing optimization algorithms. We explain the procedure in the general framework of deterministic minimization based on descent methods.

The paper ends with three examples. First we consider a simple situation featuring what we mean by robust optimization. This example shows how the approach compares with optimization after noise removal and also with momentum-based multicriteria optimization. Then, we consider the application of the algorithm to an inverse problem involving a distributed fluid mechanics model. In this case the calculation complexity is a serious issue. This makes the use of a momentum-based minimization, or any other statistical approach, to account for parameters uncertainties after their propagation through the simulation chain, unrealistic. It is shown 
that an admissible optimization problem is no longer admissible in the presence of the uncertainties on the control parameter suggesting a redefinition of the control space. This final point naturally introduce the idea of the cost of making a given uncertain system, but deterministically designed, reliable. The paper ends with a large shape optimization problem for an aircraft. This example shows the importance of including VaR information all along the design iterations leading to final shapes differences not necessarily correlated with the uncertainty distributions.

\section{VALUE AT RISK}

In financial engineering, the Value at Risk (VaR) is a widely used risk measure of the risk of loss on a given asset [14]. It defines, for a given probability level $(0<\alpha<1)$ and time horizon (typically one day), a threshold value for the loss $X$ on the asset:

$$
\operatorname{VaR}_{\alpha}=\inf \{l \in \mathbb{R}: P(X>l) \leqslant 1-\alpha\} .
$$

This is illustrated in figure 1 where the areas filled worth $\alpha$. We would like to use this concept during design knowing the PDF of the uncertainties on manufacturing realizations. During optimization a given control parameter $x$ represents, with a confidence level of $\alpha$, the interval $\left[X+\mathrm{VaR}_{\alpha}^{-}, X+\mathrm{VaR}_{\alpha}^{+}\right]$around $X$ the 'deterministic' value of that parameter and $\mathrm{VaR}_{\alpha}^{-} \leqslant 0 \leqslant \mathrm{VaR}_{\alpha}^{+}$. One expresses then possible deviations from $X$ via two PDF and the corresponding values at risk. One can make the hypothesis that the upper and lower bounds of the variations are symmetric, in which case $\mathrm{VaR}_{\alpha}^{-}=-\mathrm{VaR}_{\alpha}^{+}$. This is typically the case when uncertainties on a parameter follow a Gaussian distribution. We do not address any time issue here and make the hypothesis that the VaR is stationary. If the manufacturing process improves with time, the deviations due to manufacturing uncertainties decrease with time. On the other hand, one could account for possible wears of mechanical parts over time. These situations can be interpreted as the PDF tails getting thinner for the former and thicker for the later cases with time.

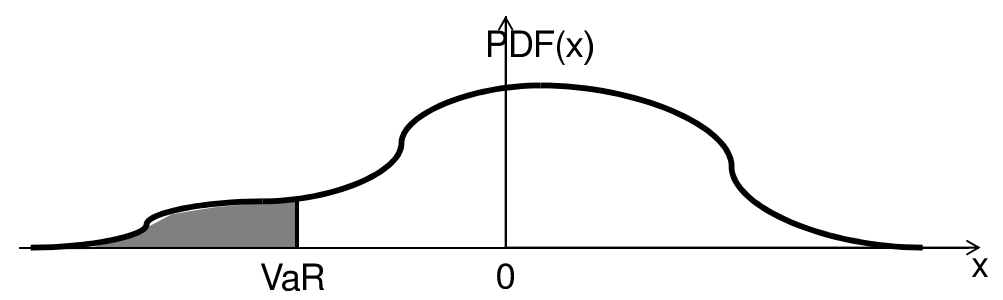

FiguRE 1. Value at risk for a general PDF. The colored area worths $\alpha$.

For the sake of simplicity we consider the context of Gaussian probability density functions for which values at risk are explicitly known: $\mathrm{VaR}_{0.99}=2.33$ and $\mathrm{VaR}_{0.95}=$ 1.65 for $N(0,1)$ and $\operatorname{VaR}_{\alpha}(N(0, \sigma))=\sigma \operatorname{VaR}_{\alpha}(N(0,1))$.

In the general case, the PDF of the uncertainties are known before optimization and so are their VaR after a numerical integration. For situations where uncertainties 
are expressed through intervals of confidence or margins, in what follows the VaR can be simply replaced by the extreme values.

\section{CONTROLLABILITY AND GLOBAL OPTIMIZATION}

Consider the minimization of a functional in finite dimension $J(x) \in \mathbb{R}, x \in \mathbb{R}^{n}$. We suppose the problem admissible (i.e. there exists at least one solution $x_{m}$ to the problem: $J\left(x_{m}\right)=J_{m}$, where $J_{m}$ is the infimum of $\left.J(x)\right)$. In practice $J_{m}$ is obviously unknown. We assume it is known for the justification of our argument.

Consider the following system:

$$
\frac{d x}{d t}=-d(x(t)), \quad x(0)=x_{0},
$$

where $d$ indicates a search direction vanishing at all critical points of $J(x)$. Finding a global minimizer for $J$ solving (1) means finding $x(T)$ for finite $T$ such that

$$
\frac{d x}{d t}=-d(x(t)), \quad x(0)=x_{0}, \quad J(x(T))=J_{m} .
$$

This is an over-determined boundary value problem. To remove this over-determination one shall consider the following second order system:

$$
\frac{d^{2} x}{d t^{2}}+\frac{d x}{d t}=-d(x(t)), \quad x(0)=x_{0}, J(x(T))=J_{m}
$$

In practice the final condition is not aimed at being exactly realized. This is even more obvious in presence of uncertainties. Rather, one would like, for a given precision $\delta$ in the functional, to build at least one trajectory $\left(x(t), 0 \leqslant t \leqslant T_{\delta}\right)$ passing for finite $T_{\delta}$ in the ball $B_{\delta}\left(x_{m}\right)$. This formulation permits to naturally accounts for uncertainties in the definition of $\delta$ using the following controllability theorem (given in [6] for (3) with $d=\nabla J$ ) as theoretical background.

Theorem: Let $J: \mathbb{R}^{n} \rightarrow \mathbb{R}$ be a $C^{2}$-function such that $\min _{\mathbb{R}^{n}} J$ exists and is reached at $x_{m} \in \mathbb{R}^{n}$. Then for every $\left(x_{0}, \delta\right) \in \mathbb{R}^{n} \times \mathbb{R}^{+}$, there exists $\left(\sigma, T_{\delta}\right) \in$ $\mathbb{R}^{n} \times \mathbb{R}^{+}$such that the solution of the following system:

$$
\frac{d^{2} x}{d t^{2}}+\frac{d x}{d t}=-\nabla J(x(t)), t \geqslant 0, x(0)=x_{0}, \frac{d x}{d t}(0)=\sigma,
$$

passes at time $t=T_{\delta}$ into the ball $B_{\delta}\left(x_{m}\right)$.

In the presence of uncertainties, the target ball $B_{\delta}\left(x_{m}\right)$ is replaced by a 'givenrisk' area as sketched in figure 2. This area, denoted $x_{m}+V a R$, obviously contains $B_{\delta}\left(x_{m}\right)$ and is the union of all the risk ellipsoids for all the points in $B_{\delta}\left(x_{m}\right)$ :

$$
x_{m}+\operatorname{VaR}=\cup_{x \in B_{\delta}\left(x_{m}\right)}\{x+\operatorname{VaR}(x)\} .
$$

When the uncertainty PDF is uniform over the admissible domain, it is enough to consider the ellipsoids for the points on the boundary of $B_{\delta}\left(x_{m}\right)$ :

$$
x_{m}+\operatorname{VaR}=\cup_{x \in \partial B_{\delta}\left(x_{m}\right)}\{x+\operatorname{VaR}(x)\},
$$

where $x+\operatorname{VaR}(x)$ is a local domain around a point $x$ :

$$
x+\operatorname{VaR}(x)=\left\{y: x(i)+\operatorname{VaR}_{\alpha}^{-}(x(i)) \leqslant y(i) \leqslant x(i)+\operatorname{VaR}_{\alpha}^{+}(x(i)), i=1, . ., n\right\} .
$$


Over-solving: This is a natural introduction of the concept of over-solving as two control points in the area described above cannot be discriminated due to uncertainties: once in this area, it is useless to look for a better optimum, even if the required accuracy $\delta$ is not achieved. We discuss this issue in section 4 .

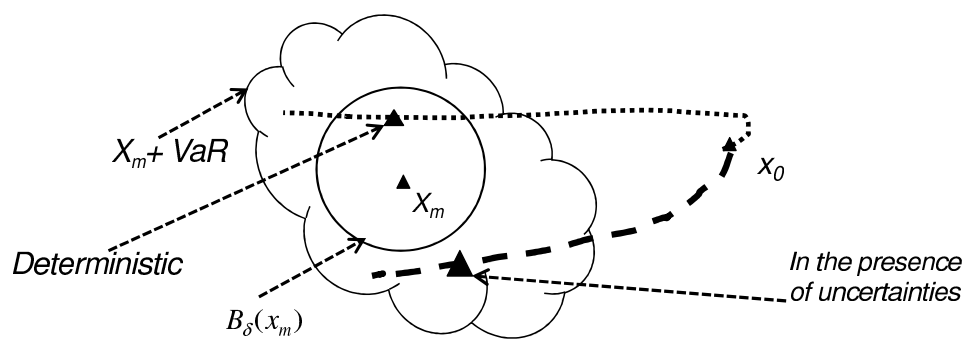

FiguRE 2. Examples of trajectories passing in $B_{\delta}\left(x_{m}\right)$ in the absence of uncertainties and the same in their presence. The admissible solution area in the latter case is a confidence subdomain defined from the knowledge of the 'local' value at risk on the parameters. Of course, the trajectories are not necessarily the same in the presence of uncertainties.

Algorithmic considerations This theorem is insightful for the derivation of global minimization algorithms as discrete forms of our second order system. However, most minimization algorithms are discrete forms of first order systems such as (1). Still shooting technics can be used to redefine the initial condition for them and improve their global search features as described in $[2,13]$.

\section{OPTIMIZING WITH THE VAR}

Now, let us introduce uncertainty information into minimization iterations through the VaR concept. For the sake of simplicity, we consider an explicit discrete form of (1) where one takes optimal descent steps along $-d$ ( $d$ can be sophisticated or simply $\nabla J)$ :

$$
\left\{\begin{array}{l}
x_{0}=\text { given, } \\
\text { for } n \leqslant N \\
\text { Evaluate } d \text { and break if }\|d\| \leqslant T O L, \\
\rho_{\text {opt }}=\text { Argmin }_{\rho \in \mathbb{R}_{*}^{+}}\left\{J\left(x_{n}-\rho d\right)\right\} \\
x_{n+1}=x_{n}-\rho_{\text {opt }} d .
\end{array}\right.
$$

Uncertainties on control parameters can be introduced in this algorithm mimicking a collaborative work in industry when iterating on the design. Knowing the PDF of these uncertainties, a muti-dimensional design point is only known with some confidence level on each of its components $x_{n+1}(i)$. We denote this confidence interval $\left[x_{n+1}(i)+\operatorname{VaR}_{\alpha}^{-}\left(x_{n+1}(i)\right), x_{n+1}(i)+\operatorname{VaR}_{\alpha}^{+}\left(x_{n+1}(i)\right)\right]$. It would make sense to consider a worst-case scenario defining a correction $W$ to $x_{n+1}$ where each component worths $\operatorname{VaR}_{\alpha}^{-}\left(x_{n+1}(i)\right)$ or $\operatorname{VaR}_{\alpha}^{+}\left(x_{n+1}(i)\right)$ and defined in order to maximize $<d, W>$, the 


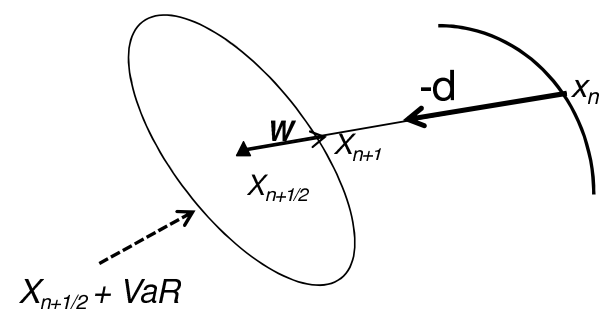

FiguRE 3. Sketch of a 'worst-case' correction given in iteration (6) and based on the knowledge of 'local' value at risk defining a 'risk' subdomain.

scalar product between $d$ and $W$. This modification should also take place in the definition of $\rho_{\text {opt }}$. This quantitative 'risk' subdomain is sketched in figure 3 (denoted below by $x+\operatorname{VaR}(x)$ for a given point $x$ ). One makes the hypothesis that the $\mathrm{VaR}$ is a priori known but, may be, not necessarily uniform over the parameter space. However, the uncertainty on a given parameter is kept unchanged during optimization. Also, the control parameters are supposed independent from each other (i.e. no correlation between control parameters components).

The minimization iterations (5) becomes:

$$
\left\{\begin{array}{l}
x_{0}=\text { given, } \\
\text { for } n \leqslant N \\
\text { Evaluate } d \text { and break if }\|d\| \leqslant T O L, \\
\rho_{\text {opt }}=\operatorname{Argmin} \\
\text { where } V=\operatorname{Rrgmax}_{v \in \operatorname{VaR}\left(x_{n}-\rho d\right)}\left\{J\left(x_{n}-\rho d+V\right)\right\}, \\
x_{n+1 / 2}=x_{n}-\rho_{o p t} d, \\
W=\operatorname{Argmax}_{v \in \operatorname{VaR}\left(x_{n+1 / 2}\right)}\{<d, v>\}, \\
x_{n+1}=x_{n+1 / 2}+W, \\
\text { break if }\left(\left\|x_{n+1 / 2}-x_{n}\right\| \leqslant\|W\|\right) .
\end{array}\right.
$$

It is important to notice that the maximization sub-problems do not involve any evaluation of the functional and are purely geometric. The algorithm may stop before in the deterministic situation as one has an extra stopping criterion which is to avoid over-solving as we will see in the numerical examples. It suggests that continuing minimization iterations is useless when the variations fall below the uncertainty level. Therefore, there is no guarantee that the first order optimality condition is achieved for the VaR-based design. Also, in the presence of several local minima the outcome of the deterministic and the VaR-based designs can be totally different because of the deviation introduced by the VaR corrections and the fact that these have been introduced during the optimization iterations.

\section{ENLARGING THE CONTROL SPACE}

A natural desire is to remove or limit the impact of the uncertainties of the design parameter on the design. We would like to proceed increasing the size of the control space introducing new uncertain design parameters in order for the outcome of 
this enlarged uncertain design to recover the behavior of the deterministic one. In other words, we make the assumption that an uncertain system will require more monitoring in order to perform as a deterministic one. Of course, one needs to minimize this extra control effort. Introducing new control variables vector $y$, let us rewrite our functional $J(x)$ as $\hat{J}(x, y) \in \mathbb{R},(x, y) \in \mathbb{R}^{n+m}$. One would like to keep $m$ as small as possible. As previously, both $x$ and $y$ are uncertain with known PDF.

We proceed with deterministic optimization using algorithm (5) and optimization including the VaR informations using (6). Let us denote the corresponding results by $x^{*}$ and $\tilde{x}^{*}$ written in short as:

$$
x^{*}=\operatorname{Argmin}_{x} J(x) \text { and } \tilde{x}^{*}=\operatorname{Argmin}_{x} J(x+\mathrm{VaR}) .
$$

$x^{*}$ and $\tilde{x}^{*}$ can be quite different as we will see in our examples.

We assume the deterministic design is the best solution one could expect. We called the corresponding functional value $J_{m}$ in section 3 and if the deterministic optimization problem is admissible and fully solved, one should have $J\left(x^{*}\right)=J_{m}$. Also, because the uncertainties only degrade the performance of a system (because otherwise $x^{*}$ would not be an optimum) one has $J\left(\tilde{x}^{*}\right)>J_{m}$. Now, to remove the impact of the uncertainties on the design the idea is to solve alternatively:

$$
\hat{x}^{*}=\operatorname{Argmin}_{x} \hat{J}\left(x, \hat{y}^{*}\right), \hat{y}^{*}=\operatorname{Argmin}_{y} \hat{J}\left(\hat{x}^{*}, y\right) \text {. }
$$

Eventually, one would like to have $\hat{J}\left(\hat{x}^{*}, \hat{y}^{*}\right)=J_{m}$. In practice, we take sequential steps for the solution of the two optimization problems looking for a Nash equilibrium [18]:

$$
\left\{\begin{array}{l}
\left(\hat{x}_{0}, \hat{y}_{0}\right)=\text { given, } \\
\text { for } n \leqslant N \\
\left\{\begin{array}{l}
\text { Evaluate } d=d\left(\hat{J}\left(\hat{x}_{n}, \hat{y}_{n}\right)\right) \text { and break if }\|d\| \leqslant T O L, \\
\rho_{\text {opt }}=\operatorname{Argmin}_{\rho \in \mathbb{R}_{*}^{+}}\left\{\hat{J}\left(\hat{x}_{n}-\rho d+V, \hat{y}_{n}\right)\right\} \\
\text { where } V=\operatorname{Argmax}_{v \in \operatorname{VaR}\left(\hat{x}_{n}-\rho d\right)}\{<d, v>\}, \\
\hat{x}_{n+1 / 2}=\hat{x}_{n}-\rho_{\text {opt }} d, \\
W=\operatorname{Argmax}_{v \in \operatorname{VaR}\left(\hat{x}_{n+1 / 2}\right)}\{<d, v>\}, \\
\hat{x}_{n+1}=\hat{x}_{n+1 / 2}+W \\
\text { break if }\left(\left\|\hat{x}_{n+1 / 2}-\hat{x}_{n}\right\| \leqslant\|W\|\right),
\end{array}\right. \\
\left\{\begin{array}{l}
\text { Evaluate } d=d\left(\hat{J}\left(\hat{x}_{n+1}, \hat{y}_{n}\right)\right) \text { and go to the next iteration if }\|d\| \leqslant T O L, \\
\rho_{\text {opt }}=\operatorname{Argmin}_{\rho \in \mathbb{R}_{*}^{+}}\left\{\hat{J}\left(\hat{x}_{n+1}, \hat{y}_{n}-\rho d+V\right)\right\}, \\
\text { where } V=\operatorname{Argmax}_{v \in \operatorname{VaR}\left(\hat{y}_{n}-\rho d\right)}\{<d, v>\}, \\
\hat{y}_{n+1 / 2}=\hat{y}_{n}-\rho_{o p t} d, \\
W=\operatorname{Argmax}_{v \in \operatorname{VaR}\left(\hat{y}_{n+1 / 2}\right)}\{<d, v>\}, \\
\hat{y}_{n+1}=\hat{y}_{n+1 / 2}+W
\end{array}\right.
\end{array}\right.
$$

The stopping criterion in (7) is the same as in (6) and only involves $\hat{x}$ and not the control variable $\hat{y}$ which has only been introduced to reduce the effect of the uncertainties on the design and improve the reliability of the system [15]. We think that it is important to keep this hierarchy between the design parameters in order to be able to downgrade a system in term of manufacturing cost. For instance, when a customer finds excessive the extra cost of having $y$ active. We show in section 7 the effect of this enlargement of the design space on an inverse problem. 


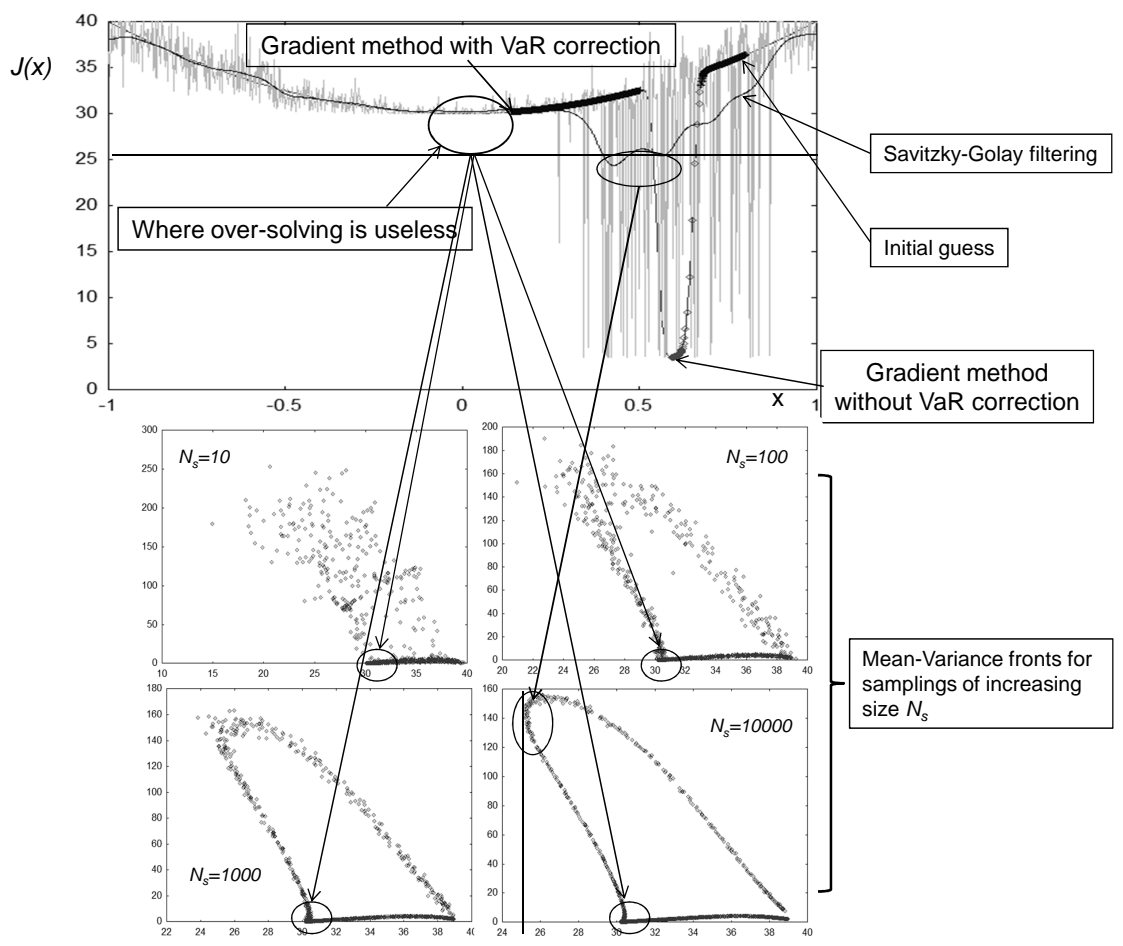

FIgURE 4. A deterministic situation can appear extremely noisy only due to uncertainties in one parameter (example in section 6) for $J(x)=30+10 x^{2}-30 \exp \left(-10^{5}(x-0.6)^{4}\right)$ on $-1 \leqslant x \leqslant 1$. Here uncertainties in the position $x$ makes that larger deviations in the functional appear near the global minimum which is therefore non-robust. One shows optimization paths without and with the VaR-based correction starting from the same initial value. The correction discriminates between the two minima in term of robustness. The approach can be linked to multicriteria 'momentum-based' optimization. Lower pictures show Pareto fronts for the mean and variance obtained with uniform samplings of increasing size $N_{s}$. Optimization of a filtered functional gives here non-robust solutions.

\section{AN EXAMPLE OF ROBUST vs. GLOBAL OPTIMIZATION}

Let us first illustrate our approach for a steepest descent algorithm where $d=\nabla J$. Consider the minimization of $J(x)=30+10 x^{2}-30 \exp \left(-10^{5}(x-0.6)^{4}\right)$ on $-1 \leqslant$ $x \leqslant 1$. The value of the control parameter is given up to an uncertainty following $\varepsilon=N(0,0.4)$. The functional has two minima. The global minimum is non-robust. One can see that a same perturbation in the control parameter creates much larger deviations around this optimum (figure 4-top). This is clearly not suitable from an industrial point of view. The second minimum (at the origin) appears, on the other hand, being robust. Our approach permits to discriminate between these minima. Also, even if this configuration is more robust, one sees that one should not over-solve near this minimum because of the uncertainties. Figure 4 indicates such a region 
where, due to uncertainties, it is impossible to discriminate between the different configurations.

6.1. Link with a multicriteria momentum-based interpretation of robust optimization. Even if this is something we would like to avoid because of its computational complexity, suppose one can propagate the uncertainty through the simulation chain and obtain a set of realizations. Then, an interesting interpretation of our approach comes when linked to multicriteria 'momentum-based' minimization which is one classical way to robust optimization involving the different momentum of the functional (e.g. mean and variance):

$$
\min _{x \in[-1,1]} \mathbb{E}(J(\tilde{x})) \text {, such that } \mathbb{E}\left(J^{2}(\tilde{x})\right)-\mathbb{E}^{2}(J(\tilde{x})) \leqslant T O L,
$$

where, as we said, $\tilde{x}=x+\varepsilon(x)$ with the PDF of $\varepsilon(x)$ known. We previously showed how to use a controllability result for a second order dynamic system such as (3) to reach points on general Pareto fronts, not necessary convex $[1,6]$. Solving this multicriteria problem is therefore possible but complexity will remain an issue.

The present approach avoids the sensitivity of the minimization to the convergence of the different momentum. Working with these quantities permits however another qualification of a robust optimum. Figure 4 shows four different Pareto fronts for the mean and variance of realizations based on uniform samplings of size $N_{s}=10,100,1000$ and 10000. As expected, one sees that convergence to a fully established front needs much larger effort in regions of large variance. On the other hand, the region of low variance is well identified, even with the poorest sampling. It corresponds to the optimum found after the introduction of our robustness concept through the VaR. This suggests a way to discriminate between minima in term of robustness using different samplings in a Monte Carlo method: the robust optimum is the one corresponding to the first stable region in the mean-variance front.

Another widely used approach in engineering involves reduced order models to make affordable optimization under uncertainty. The reduced order model can be, for instance, based on the assimilation (e.g. by least square projections) by a polynomial functional of high fidelity simulations. This has as a consequence to filter the noisy functional and obtain a smooth one $\bar{J}(x)$ to minimize. Figure 4 also shows such an outcome using the same descent method for a functional built using a SavitzkyGolay filter $[16,17]$ based on a local least-square assimilation of the noisy functional by a piecewise cubic spline model. One sees that new local minima appear and also the plausible optimum found by the minimization procedure (corresponding to $24 \leqslant \bar{J}(x) \leqslant 26$ ) belongs to regions of high variance which cannot be considered as robust. In other words, one should take great care when permuting filtering or model reduction and minimization operations. Today, for minimization under uncertainty, industry mostly relies on reduced order models. This simple example shows that this is not necessarily reliable.

\section{AN INVERSE PROBLEM WITH FLUIDS}

The problem consists of defining injection velocities and directions (the independent parameters and denoted $x$ below) in several points in the domain in order to recover a given pollutant target distribution as shown in figure 5 . We need to minimize $J(x)=\left\|c(\vec{u}(x))-c_{\text {target }}\right\|$, where $\|\cdot\|$ is a discrete $L^{2}$ norm over the calculation 
domain. The pollutant $c$ is solution of an advection-diffusion problem by a flow field $\vec{u}(x)$. The field is solution of a reduced order flow model [19] which assimilates prescribed injection velocities solution of the optimization problem. $\vec{u}(x)$ can also be solution of a PDE based flow model (e.g. Navier-Stokes equations) forced through these injections. The physical model is not central to the paper, so it is not described here. What is important is that this assimilation cannot be exact as there is no guarantee that the injections scenarios proposed by the optimization procedure are solution of the chosen flow model. Therefore, to reduce this source of error the injection points are located distant from each other.

Here, our optimization procedure is used for the definition of five different injection devices over the domain (see figure 5). Each injection is defined by its angle and flow rate. This is therefore an optimization problem in dimension ten. Also, as in any industrial processes, another source of uncertainty is due to practical realization and possible errors on the injection angles for instance. To introduce our VaR correction, we suppose we know a Gaussian PDF model for the uncertainties on each of the injection devices. Of course, there is no limitation in considering other probability density functions as long as these are a priori known with their VaR computed by a numerical integration.

The complexity of the problem makes that a 'momentum-based' minimization would be quite expensive.

Figure 5 shows that without uncertainty the minimization problem is admissible in the sense that one can find a given control distribution which permits to recover the target distribution $c_{\text {target }}$. In the presence of uncertainties, our worst-case analysis leads to a different injection distribution, and therefore flow pattern. In other words, the optimization problem is not anymore admissible. This indicates that in such cases one should modify the control space in purpose. But, the solution appears being more robust in the sense that it has been reached earlier in the optimization procedure and kept unchanged. It should be seen as a configuration which is not optimal but, because of the uncertainties in the control devices, one might not be able to get much better. The interesting point is that both optimizations required the same calculation effort and this was our main motivation in this work.

Now, to reduce the effect of the uncertainties of the design parameters, one proceeds to an enlargement of the design space as described in section (5) and uses algorithm (7) after adding one and then two injection devices (those are in variable $y$ ). Both injectors are supposed having the same level of uncertainty than the previous five. The dimension of the control space becomes 12 then 14 . The positions of the injection devices is arbitrary and far from those defined through variable $x$. Obviously, the positions of the new injectors could have been optimized as part of the variable $y$. Figure 5 also compares the two previous convergence histories in dimension 10 to those of $\hat{J}(x, y)$ in dimension 12 and 14 . One can see that one extra injector permits to recover the deterministic design even in the presence of uncertainties. With two injectors the recovery is faster. This kind of information is interesting in industrial design as it gives an indication on the extra cost for making a given design reliable: in the sense of having a behavior compliant with what defined for the deterministic system. 


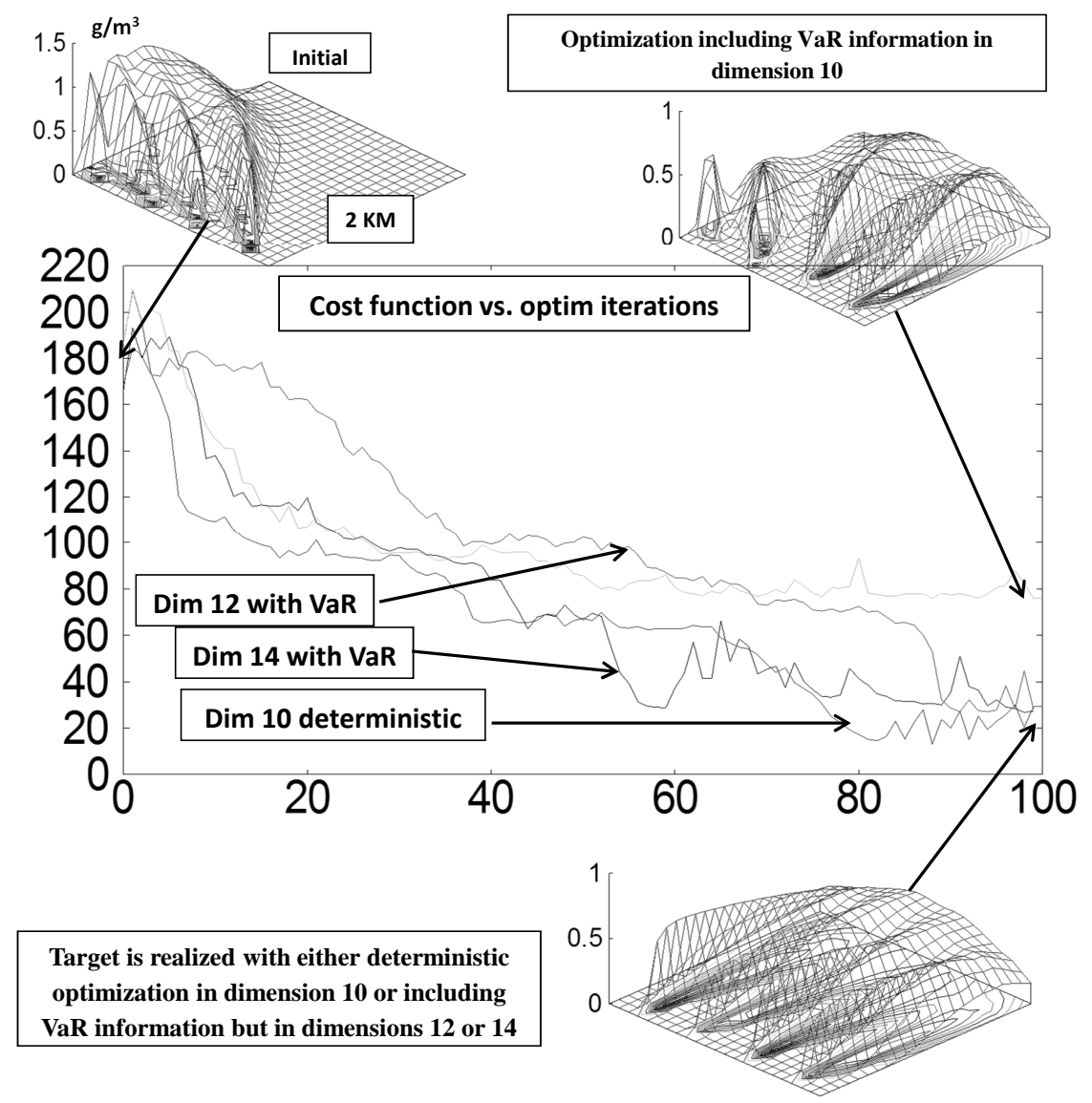

Figure 5. The aim is to recover the flow which permits a target passive scalar $c(\vec{u}(x))$ distribution. A pure deterministic optimization recovers the target quite well but not when including uncertainties on the injection devices. Introducing new control devices permit to recover the target distribution. Hence, one can control/remove the impact of the uncertainties on the design increasing the size of the control parameter space. Here, one extra injection device was sufficient but with two the target design is reached earlier in the optimization process.

\section{FULL AIRCRAFT SHAPE OPTIMIZATION}

The third example concerns a shape optimization problem for a full aircraft in transonic cruise condition. We would like to include a priori known VaR informations on the shape.

Let us briefly describe the shape optimization procedure $[2,20]$. A full description would be obviously too long. The discussion concentrates then on the introduction of the VaR concept in the design loop. Our design uses the linearization by an adjoint approach of a direct calculation loop linking the vector of control parameters $x$ (here 
of dimension 5000) to a functional $J$ :

$$
J(x): x \rightarrow q(x) \rightarrow U(q(x)) \rightarrow J(x, q(x), U(q(x))) .
$$

$x$ is a CAD-free parameterization of the shape (CAD: Computer Aided Design) [20] which does not require a priori local regularity assumptions on the shape as it is implicitely the case in CAD-based shape definitions. $q(x)$ denotes auxiliary unstructured mesh related geometrical quantities and $U(q(x))$ flow variables, solution here of the Reynolds averaged Navier-Stokes equations and a two equations turbulence model [22]. The derivative $\nabla_{x} J$ is computed by automatic differentiation in reverse mode using tapenade [21] and we have optimized by hand the produced reverse mode code for steady flow adjoint solutions in order to minimize memory requirements in reverse loops [2]. The optimization procedure requires an initial shape parameterization and a priori Value at Risk information for this three dimensional shape as a local vector in $\mathbb{R}^{3}$ (i.e. of size $5000 \times 3$ ) representing the variability on each of the coordinate of the shape at each point of the CAD-free parameterization. The initial shape and $\|V a R\|$ contours are shown in figure 6. We assume larger uncertainties along the wings and increasing spanwise.

The optimization aims at maximizing $C_{l}$ the lift and minimizing $C_{d}$ the drag coefficient:

$$
J(x, q(x), U(x))=-\frac{C_{l}}{C_{l}^{0}}+\frac{C_{d}}{C_{d}^{0}},
$$

where superscript 0 indicates the initial shape. Figure 6 shows the shapes obtained by algorithms (5) and (6). The lift to drag ratio is higher for the deterministic design. Convergence histories in figure 6 are for the aerodynamic coefficients normalized by the values on the initial shape. Because informations on the $\mathrm{VaR}$ were accounted for during the design iterations and not only at the end of the procedure, shapes differences are not necessarily where uncertainties are larger. This indicates probable presence of local minima for this problem. This can be seen when one switches off the VaR correction after 40 iterations of optimization in (6). Figure 7 shows shape deformations with respect to the initial shape with the deterministic and the VaRbased then deterministic optimizations. The two shapes have the same performance in term of drag but differ on the lift coefficient.

\section{CONCLUDING REMARKS}

In order to be easily integrated in engineering environments, robust optimization has been addressed in the framework of deterministic algorithms by the introduction of the Value at Risk concept. This permits to quantify our confidence on the optimal solution without any sampling of the control space. We deliberately work in a global optimization framework because these considerations permit to discriminate between minima and only consider robust solutions. Also, one clear advantage of this approach is that one remains in the deterministic framework with supposed sufficient regularity where the gradient of the functional can be defined and computed using classical approaches such as finite differences or via an adjoint. The approach also naturally features the need to avoid over-solving introducing a stopping criteria based on the ratio of variations during optimization and local uncertainties. It has been shown through numerical examples that this VaR-based correction can be linked to momentum-based optimization. The solution by the approach appears being the robust optimum in the mean-variance front generated through Monte 
Carlo simulations with increasing sample sizes. Introduction of new control devices monitoring the impact of the uncertainties has been discussed in order to give an indication on the cost of making an uncertain system reliable. Finally, the importance of including the VaR information during the design iterations has been discussed through shape optimization of a full aircraft.

\section{REFERENCES}

[1] Pareto V.Manuale di Economia Politica, 1906, Societa Editrice Libraria, Milano, Italy. Translated into English by Schwier, A.S.: Manual of Political Economy, New York, Macmillan, 1971.

[2] Mohammadi B., Pironneau O. Applied Shape Optimization for Fluids (2nd Edition), Oxford Univ. Press, 2009.

[3] Schillings C., Schmidt S., and Schulz V. Efficient shape optimization for certain and uncertain aerodynamic design. Computers \& Fluids, 46(1):78-87, 2011.

[4] Bungartz, H.-J. and Griebel, M. Sparse Grids, Acta Numerica, 13, 147:269, 2004.

[5] Wan X., Karniadakis G.E., Multi-element generalized polynomial chaos for arbitrary probability measures, SIAM J. Sci. Comput. 28(3): 901-928, 2006.

[6] Mohammadi B, Redont P. Improving the identification of general Pareto fronts by global optimization. C. R. Acad. Sci. Paris, Ser. I. 2009; 347: 327-331.

[7] Jahn J. Vector Optimization: Theory, Applications and Extensions, Springer, 2004.

[8] Nocedal J., Wright S.Numerical Optimization, Springer, NY, 2006.

[9] Tarantola A. Inverse problem theory and methods for model parameter estimation, SIAM, NY, 1987.

[10] Casella G., Berger R.Statistical Inference, 2nd ed., Duxbury Press, London, 2001.

[11] Ide K., Courtier P., Ghil M., Lorenc A. Unified notation for data assimilation: operational, sequential and variational. Journal of the Meteorological Society of Japan 1997; 75(1B):181189.

[12] Conn A., Scheinberg K., Vicente L.Introduction to Derivative-Free Optimization, SIAM, NY, 2002.

[13] Ivorra B, Mohammadi B, Ramos A. semi-deterministic global optimization method and application to the control of Burgers equation. JOTA 2007; 135(1): 549-561.

[14] Jorion Ph. Value at Risk: The New Benchmark for Managing Financial Risk, McGraw-Hill, 2006.

[15] Kapur K.C., Lamberson L.R.Reliability in Engineering Design, John Wiley \& Sons, New York, 1977.

[16] Savitzky A., Golay M. Smoothing and Differentiation of Data by Simplified Least Squares Procedures, Analytical Chemistry 1964; 36(8): 1627-1639.

[17] Steinier J.,Termonia Y., Deltour J. Comments on smoothing and differentiation of data by simplified least square procedure. Analytical Chemistry 1972; 44(11): 1906-1909.

[18] Nash, J. Equilibrium points in $n$-person games. Proceedings of the National Academy of Sciences, 36(1):48-49, 1950.

[19] Brun JM., Mohammadi B. Reduced order modelling of dispersion, PDE: Modelling and Simulation, R. Glowinsky and P. Neittaanmaki Eds. Computational Methods in Applied Sciences Series, Springer; 245:259.

[20] Mohammadi, B. Pironneau, O. Shape Optimization in Fluid Mechanics, Annual Revue of Fluid Mechanics, 36-1: 255-279, 2004.

[21] Hascoet, L. Pascual, V. Tapenade 2.1 user's guide. INRIA Technical Report RT-300, 2004.

[22] Mohammadi, B. Pironneau, O. Analysis of the $k-\varepsilon$ turbulence model, Wiley, London, 1991.

Bijan Mohammadi, Université Montpellier II, Mathématiques, CC51, 34095 MontPELLIER, FRANCE

E-mail address: bijan.mohammadi@math.univ-montp2.fr 

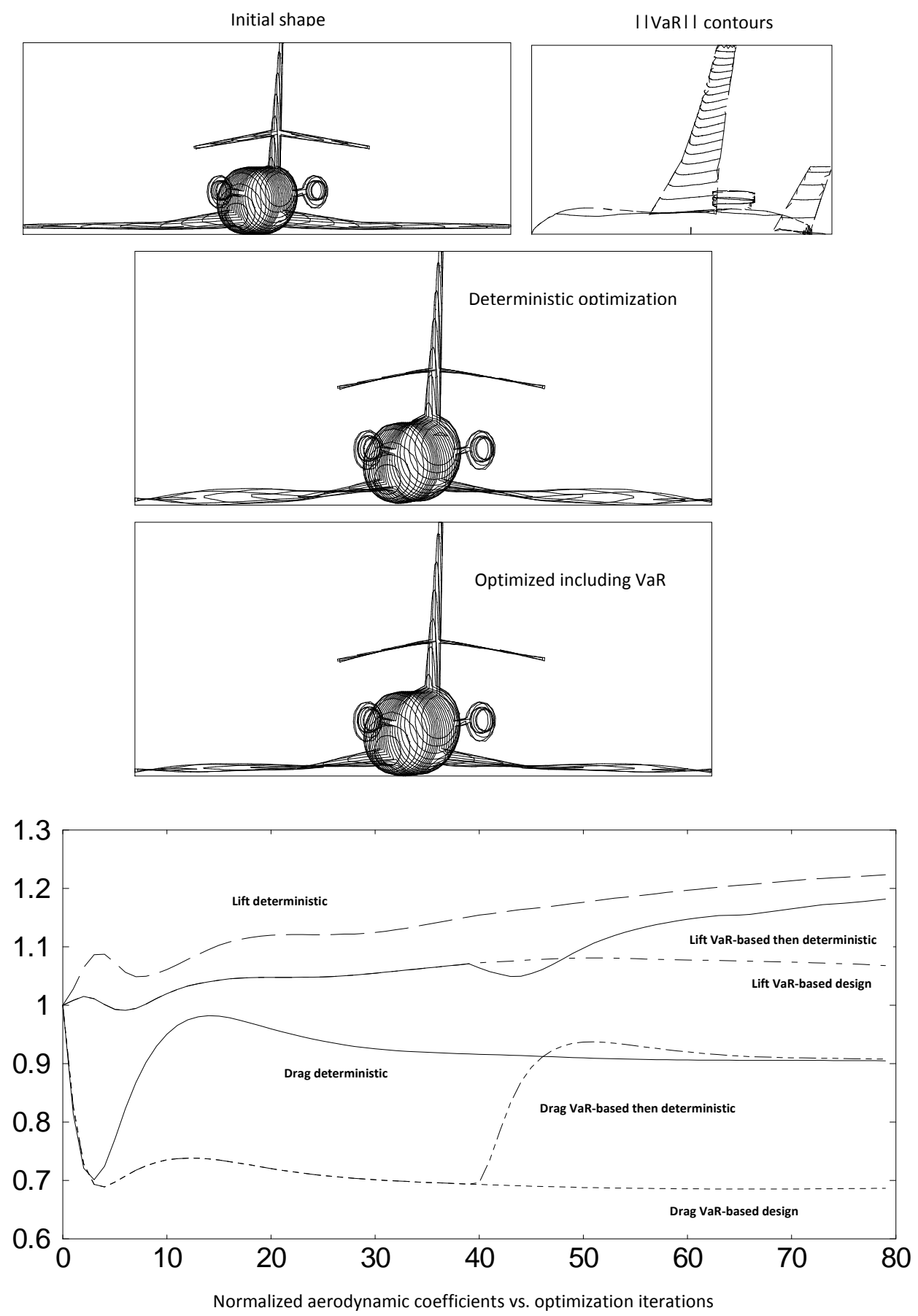

FiguRE 6. Deterministic and VaR-based shape optimizations for a full aircraft. The difference between the two shapes are not necessarily where larger uncertainties are present. Deterministic design produces higher lift to drag ration, but surprisingly, the design under uncertainty performs best in term of drag. 

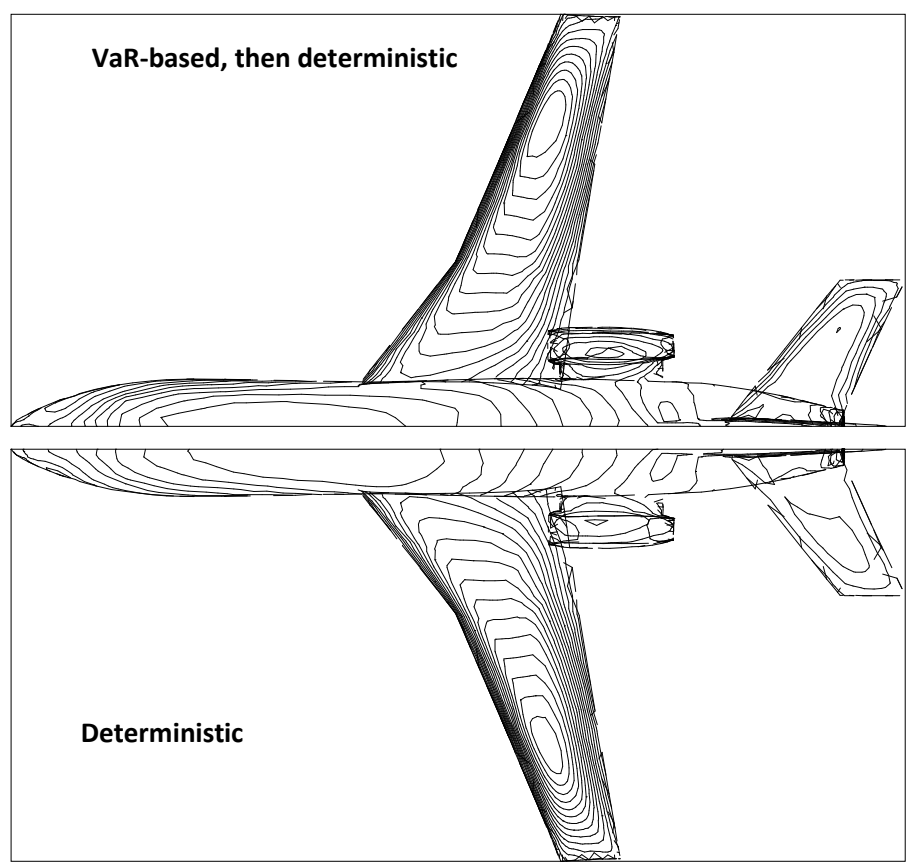

FiguRE 7. Iso-contours of deformations with respect to the initial shape with deterministic and VaR-based optimizations with the VaR correction switched off after 40 iterations of (6). 